\title{
Diphenylene iodonium interferes with cell cycle progression and induces apoptosis by modulating NAD(P)H oxidase/ROS/cell cycle regulatory pathways in Burkitt's lymphoma cells
}

\author{
YA DING $^{1 *}$, WENJUN ZHU $^{2 *}$, RUI SUN ${ }^{1}$, GANG YUAN $^{3}$, DONGSHENG ZHANG ${ }^{1}$, YUHUA FAN $^{3}$ and JIAN SUN ${ }^{1}$ \\ ${ }^{1}$ Sun Yat-sen University Cancer Center, State Key Laboratory of Oncology in South China, Collaborative Innovation \\ Center for Cancer Medicine, Guangzhou 510060; ${ }^{2}$ Guanghua School of Stomatology, Sun Yat-sen University, \\ Guangzhou 510055; ${ }^{3}$ First Affiliated Hospital, Sun Yat-sen University, Guangzhou 510080, P.R. China
}

Received October 4, 2014; Accepted December 23, 2014

DOI: 10.3892/or.2015.3726

\begin{abstract}
Infection with Epstein-Barr virus (EBV) and its encoded latent membrane protein 1 (LMP1) play oncogenic roles in Burkitt's lymphoma (BL). Flow cytometry was used to measure cellular reactive oxygen species (ROS) concentrations, and cellular lactate generation and diphenylene iodonium (DPI) cytotoxicity were determined by analyzing lactate concentrations and cell viability. We also measured $\mathrm{NAD}(\mathrm{P}) \mathrm{H}$ oxidase (NOX) activity. Reverse transcriptase PCR and qPCR assays were used to analyze LMP1 levels, and protein expression was measured by immunoblotting. In the present study, EBV was able to induce NOX activity and ROS generation in the BL cells. Inhibition of NOX activity by DPI suppressed ROS levels and elevated lactate levels. DPI treatment first resulted in a G2-M phase cell cycle arrest and then induced significant apoptosis. Immunoblot analysis demonstrated that DPI suppressed the expression of c-Myc and Cdc25A within $6 \mathrm{~h}$, which may have caused the cell cycle arrest. Collectively, these findings indicate a close relationship between EBV infection and NOX activation, permitting a deeper understanding of ROS inhibition in cell cycle regulation and providing a novel therapeutic target for BL treatment.
\end{abstract}

Correspondence to: Dr Yuhua Fan, First Affiliated Hospital, Sun Yat-sen University, Guangzhou 510080, P.R. China

E-mail: fanyuhua@mail.sysu.edu.cn

Dr Jian Sun, Sun Yat-sen University Cancer Center, State Key Laboratory of Oncology in South China, Collaborative Innovation Center for Cancer Medicine, Guangzhou 510060, P.R. China

E-mail: sunjian@sysucc.org.cn

*Contributed equally

Key words: diphenylene iodonium, cell cycle, Raji BL cells, ROS

\section{Introduction}

Burkitt's lymphoma (BL) is a malignancy of the human lymphatic system that was first described in children in Central Africa by the surgeon Denis Parsons Burkitt in 1956. The pathogenesis of both the endemic and the sporadic types of BL are closely related to impaired immunity and EpsteinBarr virus (EBV) infection (1,2). In addition to EBV, BL is also associated with a translocation involving the c-Myc and $\operatorname{IgH}$ genes, $\mathrm{t}(8 ; 14)(\mathrm{q} 24 ; \mathrm{q} 32)$ (3). The first human BL cell line, Raji, was established in 1963 and was found to integrate the EBV into its genome, leading to the discovery and isolation of this virus (4). EBV infection has been reported to contribute to BL development by increasing the lymphomagenic potential of c-Myc translocation-positive cells (5). Moreover, Lassoued et al suggested that the EBV infection of B lymphocytes and epithelial cells leads to oxidative stress that facilitates viral transformation (6). Cerimele et al also demonstrated that EBV-positive BL cells exhibit higher levels of reactive oxygen species (ROS) than EBV-negative $\mathrm{BL}$ cells and that latent membrane protein 1 (LMP1) is a major inducer of ROS (7). LMP1 is known to resemble CD40 and function as a constitutively active tumor necrosis factor receptor (TNFR) (8). Ha et al showed that the ligation and activation of CD40 produces ROS by activating the NAD $(\mathrm{P}) \mathrm{H}$ oxidase (NOX) regulatory subunit $\mathrm{p} 40^{\text {phox }}(9)$. The NOX family is an important intrinsic source of ROS that consists of seven catalytic enzymes (NOX1-5 and DUOX 1-2) and six regulatory subunits (10). Using $\mathrm{NAD}(\mathrm{P}) \mathrm{H}$ as a substrate, NOX catalyzes the conversion of oxygen to superoxide.

In cancer cells, ROS stimulate oncogenic transformation, cell proliferation, and mitochondrial malfunction (11-14), and the redox status alters the sensitivity of cancer cells to anticancer agents (15). In BL cells, an amonafide analog was demonstrated to actively inhibit cell proliferation and induce apoptosis via an ROS-mediated mitochondrial pathway (16). Rituximab was found to elevate ROS generation and sensitize BL cells to X-irradiation (17). Since multiple effective and clinically used chemotherapy agents induce excess ROS accumulation to increase their anticancer activities $(18,19)$, most researchers have focused on developing ROS-stimulating 
compounds to improve anticancer treatment outcomes $(15,20)$. However, less attention has been paid to the anticancer effects of ROS-suppressing compounds.

Scientists believe that ROS are important carcinogens (21) and that ROS-suppressing compounds can be used as chemoprevention drugs to reverse ROS-induced carcinogenesis (22). For example, polyphenols have been reported to target cyclooxygenase-2 (COX-2) and scavenge ROS in tumor cells, indicating the antitumorigenic potentials of these compounds (23). In addition to the polyphenol compounds derived from phytonutrients, microalgal products have also exhibited chemopreventative activities in cancer cells (24). The chemical compound dibenziodolium chloride (DPI), which is a potent inhibitor of NOX, is thought to significantly suppress ROS levels, and Yamaura et al reported that inhibition of NOX activity by DPI arrests melanoma cells at the G2-M phase of the cell cycle (25). Although anti-ROS compounds have been proven to prevent the development of various types of cancers, there are limited reports of their destruction of cancer cells.

In the present study, we found that EBV-encoded LMP1 was responsible for the activation of NOX and the accumulation of cellular ROS in Raji BL cells. Inhibition of EBV-activated NOX by DPI not only suppressed cellular ROS levels but also led to lactate accumulation, which first arrested the Raji cells at the G2-M phase of the cell cycle and subsequently resulted in significant apoptosis. The obvious decreases in the expression levels of c-Myc and Cdc25A within $6 \mathrm{~h}$ of DPI treatment suggest that c-Myc and Cdc25A may be responsible for DPI-induced cell cycle arrest in Raji BL cells. In this case, the ability of EBV infection to activate NOX and stimulate ROS generation represents a novel therapeutic target for BL treatment. Due to its ability to inhibit NOX-ROS-cell cycle progression, it is possible to use DPI to suppress the proliferation of BL cells.

\section{Materials and methods}

Materials. CM- ${ }_{2}$ DCF-DA was purchased from InvitrogenMolecular Probes (Carlsbad, CA, USA). DPI was purchased from Sigma-Aldrich (St. Louis, MO, USA), dissolved in dimethyl sulfoxide (DMSO) and freshly diluted in culture media before use. The final DMSO concentration was $<0.1 \%$ $(\mathrm{v} / \mathrm{v})$.

Cell lines. Raji cells, which were derived from a well-established human BL cell line with latent EBV infection, JM1 cells (an acute lymphoblastic leukemia B cell line) and NALM 16 cells (a human hematopoietic B cell line) were maintained in our laboratory in RPMI-1640 medium (Gibco-BRL, Gaithersburg, MD, USA) supplemented with $10 \%$ heat-inactivated fetal bovine serum (Thermo Scientific, HyClone, Logan, UT, USA). Cells were incubated in a humidified, $5 \% \mathrm{CO}_{2}$ atmosphere at $37^{\circ} \mathrm{C}$.

Flow cytometry. Cellular ROS concentrations were measured by incubating control or drug-treated Raji, JM1 and NALM16 cells with $1 \mu \mathrm{M} \mathrm{CM}-\mathrm{H}_{2} \mathrm{DCF}-\mathrm{DA}$ for $60 \mathrm{~min}$ followed by flow cytometric analysis using a FACSCalibur equipped with CellQuest Pro software. CM- $\mathrm{H}_{2} \mathrm{DCF}-\mathrm{DA}$ is a fluorescent probe with relative specificity for hydrogen peroxide (41). To determine the effects of the drug on the cell cycle, propidium iodide (PI) staining after $75 \%$ alcohol fixation was used, followed by flow cytometric analysis. Cell death was determined by flow cytometry after the cells were double stained with Annexin V-FITC and PI using an assay kit from BD Pharmingen (San Diego, CA, USA) in a routine manner.

Examination of cellular lactate generation. To analyze cellular lactate production, cells in the exponential growth phase were incubated with fresh medium or medium containing DPI for $24 \mathrm{~h}$. Aliquots of culture medium were then removed for analysis of lactate concentrations using a portable Accutrend lactate analyzer with a linear range of standard lactate concentrations, according to the manufacturer's recommendations (Roche, Mannheim, Germany).

Assays for DPI cytotoxicity. Cell viability was assessed using MTS assays. Raji cells were plated in 96-well culture clusters (Costa, Cambridge, MA, USA) at densities of 20,000-30,000 cells $/ \mathrm{ml}$, and serial dilutions of DPI were prepared at concentrations of $2.5-20 \mu \mathrm{M}$ using a stock solution. All experimental concentrations were prepared in triplicate. The percent absorbance of the DPI-treated cells relative to that of the DMSO-treated control cells $(<0.1 \%$ DMSO $)$ was plotted as a linear function of the DPI concentration. The antiproliferative effect of DPI on the Raji cells was measured as the percentage of viable cells relative to the DMSO-treated control cells.

NOX activity assay. DPI is widely used as an inhibitor of flavoenzymes, particularly NOX. To determine cellular NOX activity, $10 \mu \mathrm{M}$ DPI was added $4 \mathrm{~h}$ before the cells were harvested. The control and the DPI-treated NP69 and NP69-LMP1 cells were lysed in hypotonic phosphate buffer containing protease inhibitors, disrupted by sonication, and centrifuged for $10 \mathrm{~min}$ at $1,500 \mathrm{rpm}$. The supernatant containing the cytosolic and mitochondrial fractions was further ultracentrifuged at $100,000 \mathrm{x}$ g for $30 \mathrm{~min}$ at $4^{\circ} \mathrm{C}$. The resulting pellet, which contained the membranous fractions of the cytosol and mitochondria, was resuspended in buffer B [50 mM Tris (pH 7.5), 150 mM NaCl, 1 mM EDTA, and protease inhibitor cocktail (one tablet for $10 \mathrm{ml}$ of buffer)] and used for the assay. The samples were adjusted to the same protein concentration $(1 \mu \mathrm{g} / \mu \mathrm{l})$. Then, 5- $\mu 1$ aliquots of the samples were incubated with $94 \mu 1$ of phosphate buffer $(50 \mathrm{mM}$ $\mathrm{K}_{2} \mathrm{HPO}_{4}, 1 \mathrm{mM}$ EGTA, and $150 \mathrm{mM}$ sucrose) and with $1 \mu \mathrm{l}$ of 3-NAD(P)H (4 mM) for $15 \mathrm{~min}$. Before measurements were performed, $2.5 \mu \mathrm{l}$ of lucigenin $(2 \mathrm{mM})$ was added, and the lucigenin-derived chemiluminescence of the cell homogenates was assessed over $1 \mathrm{~min}$ in a 20/20 $0^{\mathrm{n}}$ tube luminometer (Turner Biosystems, Sunnyvale, CA, USA).

Small interfering RNAs and transfection. The siRNA pools for LMP1 and the non-targeting siRNAs were purchased from Thermo Scientific Dharmacon. Lipofectamine ${ }^{\mathrm{TM}} 2000$ transfection reagent (Invitrogen) was used to introduce the siRNA (final concentration of $50 \mathrm{nM}$ ) or plasmids [the ratio of DNA $(\mu \mathrm{g})$ to lipid reagent $(\mu \mathrm{l})$ was 1 to 2.5 ] into the cells. 
A

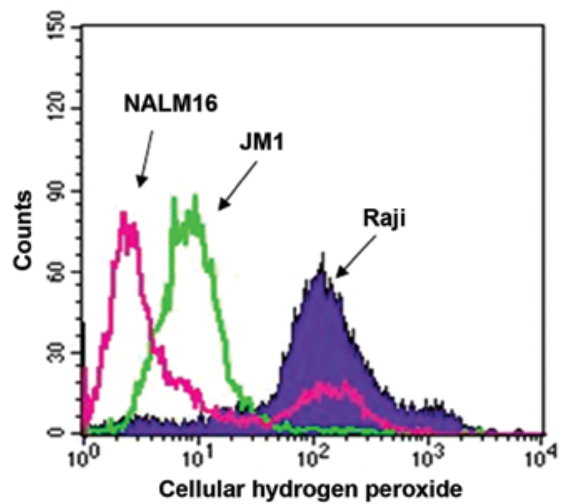

C

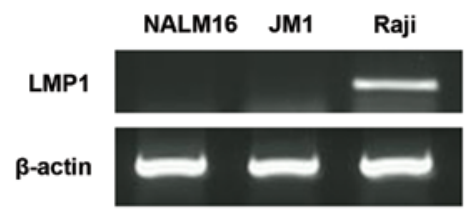

E

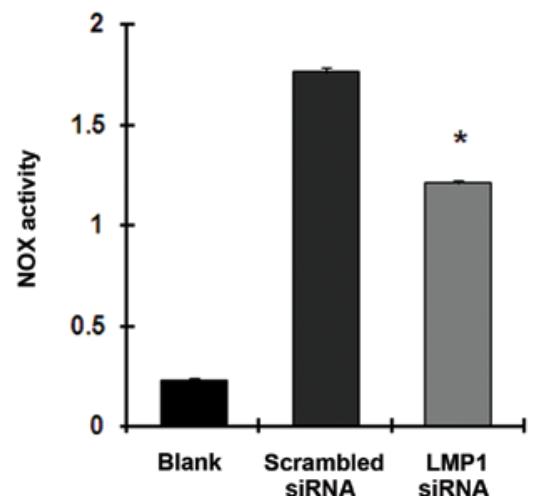

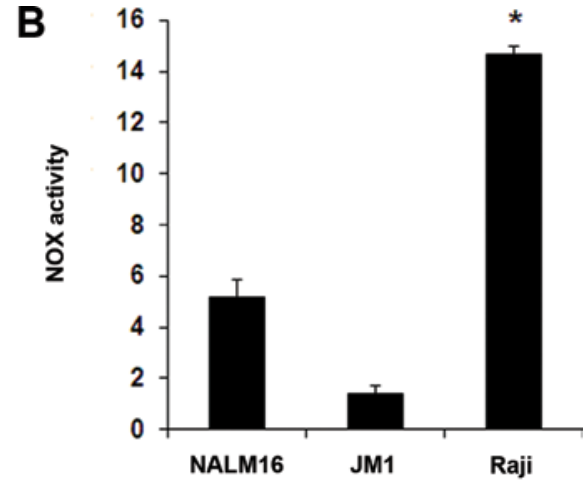

D

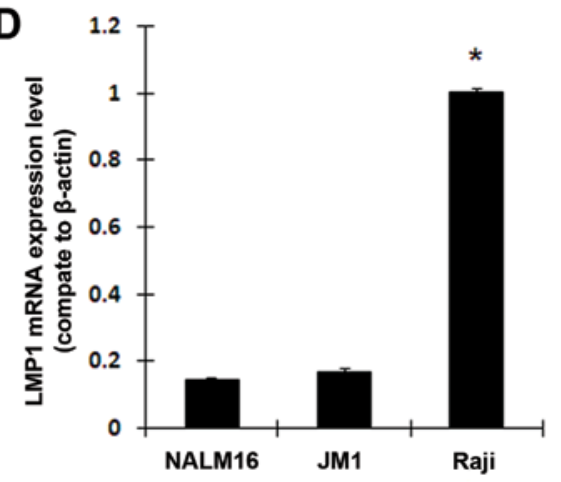

Figure 1. Epstein-Barr virus-encoded LMP1 upregulates NOX activity and ROS levels in Raji BL cells. (A) Comparison of cellular ROS concentrations in Raji, JM1 and NALM16 cells using flow cytometry and $\mathrm{H}_{2}$ DCF-DA. Each histogram is representative of three experiments (p<0.001, Raji vs. JM1 cells). (B) Comparison of NOX activity in Raji, JM1 and NALM16 cells, measured using a luminometer and lucigenin in the presence of NAD(P)H (mean \pm SD of three experiments; * $<0.01)$. (C) mRNA expression of LMP1 in NALM16, JM1 and Raji cells, measured by RT-PCR assays. $\beta$-actin served as an internal control. (D) Quantitative real-time PCR was performed to quantify mRNA expression of LMP1 in Raji, JM1 and NALM16 cells (mean \pm SD of three experiments; " $\mathrm{p}<0.001$ ). (E) The effects of LMP1 siRNA on NOX activity in Raji cells were measured using a luminometer and lucigenin in the presence of $\mathrm{NAD}(\mathrm{P}) \mathrm{H}$ (mean \pm SD of three experiments; $\left.{ }^{\mathrm{p}}<0.01\right)$. LMP1, latent membrane protein 1; NOX, NAD(P)H oxidase; ROS, reactive oxygen species; BL, Burkitt's lymphoma.

Reverse transcriptase PCR and $q P C R$ assays for LMP1. Total RNA was isolated from cells using an RNeasy Mini kit (Qiagen, Valencia, CA, USA) according to the manufacturer's instructions, and cDNA was synthesized as previously described (4). Thirty-five cycles of PCR were performed, and the primer sequences used to amplify LMP1 were as follows: forward, 5'-CGTTATGAGTGACTGGACTGGA-3' and reverse, 5'-TGAACAGCACAATTCCAAGG-3'. Quantifications of LMP1 mRNA expression in the NPC and non-cancer cells were performed by amplifications using SYBR-Green. The absolute threshold cycle values (Ct values) of LMP1 were determined using SDS software v.2.1 (Applied Biosystems). LMP1 mRNA expression was analyzed by determining its $\mathrm{Ct}$ values relative to those of $\beta$-actin.

Immunoblot analysis. Protein analyses by immunoblotting were performed as previously described (42) using primary antibodies against c-Myc (sc-764; Santa Cruz Biotechnology, Santa Cruz, CA, USA), Cdh1 (ab3242; Abcam), Chk1 (\#2345; Cell Signaling), Cdc25A (sc-97; Santa Cruz Biotechnology), DDB1 (\#5428; Cell Signaling), CDK2 (sc-163), cyclin E (sc-481) (both from Santa Cruz Biotechnology) and GAPDH (ab9489; Abcam). Total cell lysates were harvested, subjected to $12 \%$ SDS-polyacrylamide gel electrophoresis and then transferred to polyvinylidene difluoride membranes (Roche, Basel, Switzerland). Immunoblotting involved incubation with the primary antibodies followed by the addition of secondary antibodies conjugated to horseradish peroxidase (Cell Signaling) to facilitate detection. Subsequently, enhanced chemiluminescence reagent (Cell Signaling) was added to develop the blots.

Statistical analysis. All analyses for comparing the significance of the measured levels were performed using the one-way ANOVA test with SPSS 19.0 software. 
A

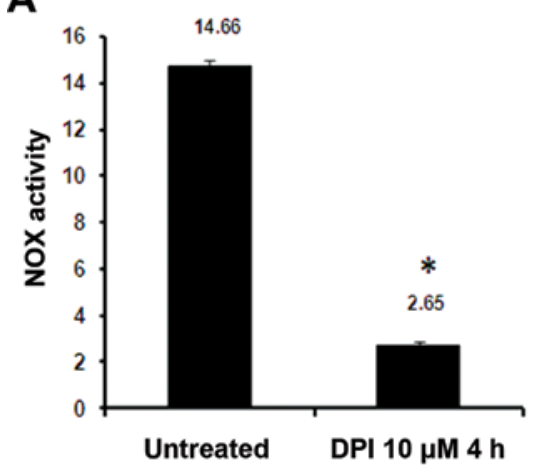

C

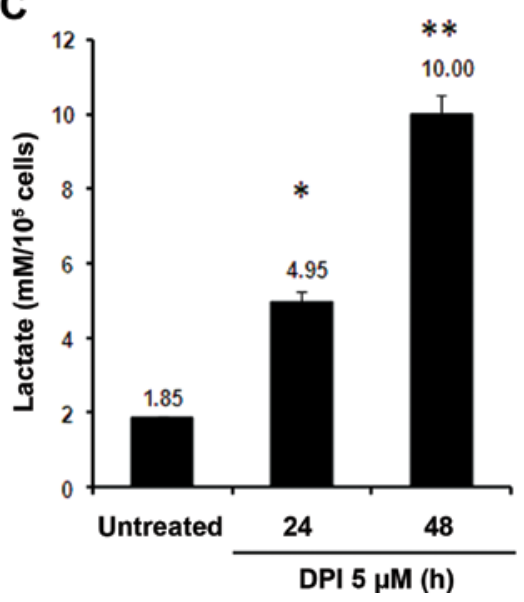

B

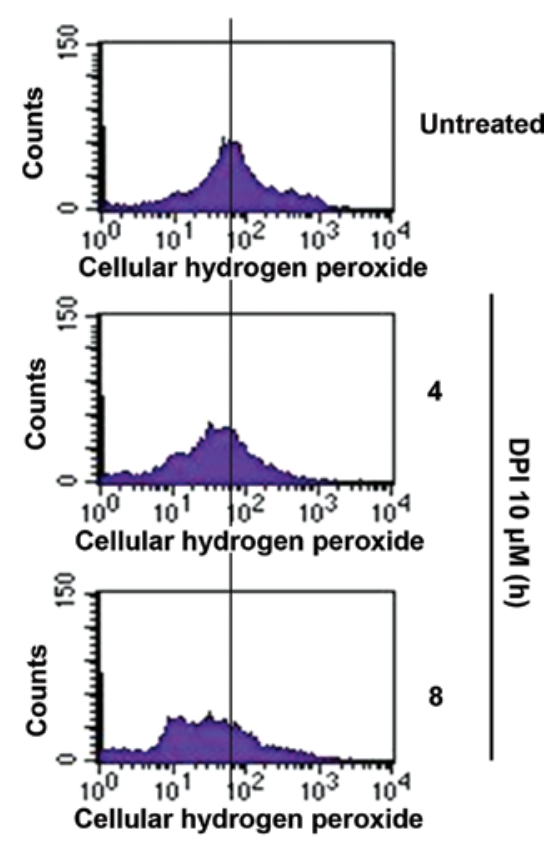

Figure 2. DPI suppresses ROS concentrations and increases lactate production in Raji cells. (A) The effects of a 4-h 10- $\mu$ M DPI treatment on NOX activity in the Raji cells was measured using a luminometer and lucigenin in the presence of NAD(P)H (mean \pm SD of three experiments; ${ }^{*}$ p $\left.<0.01\right)$. (B) The effects of 5- $\mu \mathrm{M}$ DPI treatments for 0,12 and $24 \mathrm{~h}$ on the ROS level in the Raji cells were measured using flow cytometry and $\mathrm{H}_{2} \mathrm{DCF}-\mathrm{DA}$. Each histogram is representative of three experiments. (C) The effects of 5- $\mu \mathrm{M}$ DPI treatments for 24 and $48 \mathrm{~h}$ on lactate production in the Raji cells were measured using an Accutrend lactate analyzer (mean $\pm \mathrm{SD}$ of three experiments; $\left.{ }^{*} \mathrm{p}<0.01,{ }^{* *} \mathrm{p}<0.001\right)$. ROS, reactive oxygen species; NOX, NAD(P)H oxidase.

\section{Results}

Accumulation of cellular ROS in Raji BL cells is triggered by EBV-encoded LMPI. BL is unique among the various types of $\mathrm{B}$ cell malignancies since its carcinogenesis is closely associated with EBV infection. EBV-encoded LMP1 is predominantly expressed in B cell lymphocytes latently infected with EBV. The virus-encoded nuclear protein 2 (EBNA2) and its downstream factor LMP1 have been reported to facilitate ROS production in malignant $B$ cells (7). The NOX family proteins consume NAD(P)H and transfer electrons across biological membranes to generate superoxide and ROS (26). Thus, we hypothesized that LMP1 may be able to stimulate ROS generation by activating NOX in BL cells. To test our hypothesis, we first analyzed ROS and NOX activity levels in Raji cells (from a well-established BL cell line with latent EBV infection) and in two other EBV-negative, malignant B cell lines, JM1 (an acute lymphoblastic leukemia B cell line) and NALM 16 (a human hematopoietic B cell line). As illustrated in Fig. 1A and B, the Raji cells exhibited a much higher basal ROS level (9.7- and 35.9-fold) and significantly higher NOX activity (2.9- and 10.5-fold) compared with the EBV-negative B cell lines NALM16 and JM1, respectively $(\mathrm{p}<0.01)$. Next, we detected EBV-encoded LMP1 mRNA levels by RT-PCR and qPCR. As shown in Fig. 1C and D, the expression of LMP1 mRNA was much higher in the Raji BL cells than in the JM1 and NALM16 cells. To identify the effects of LMP1 on NOX activation in the Raji BL cells, we knocked down LMP1 expression using RNAi and then analyzed NOX activity using lucigenin and a luminometer. As shown in Fig. 1E, inhibition of LMP1 expression by siRNA markedly suppressed NOX activity, suggesting that EBV LMP1 may be responsible for activating NOX enzyme activity as well as the generation of excessive ROS in the EBV-positive Raji BL cells.

\section{Inhibition of NOX activity by DPI suppresses cellular ROS} accumulation and stimulates lactate generation. Over the past decade, most studies have focused on evaluating ROS-stimulated compounds, investigating their effects on the destruction of cancer cells since excessive ROS accumulation is thought to result in the apoptosis of these cells $(15,20)$. However, little attention has been paid to the application of novel compounds that suppress cellular ROS levels in anticancer treatments. In the present study, we found that the small-molecular-weight compound DPI was able to effectively inhibit NOX activity (by $\sim 82 \%$, Fig. $2 \mathrm{~A}$ ) in the Raji cells. Since the aforementioned findings suggest that LMP1-activated NOX is responsible for stimulating ROS generation in Raji BL 
A

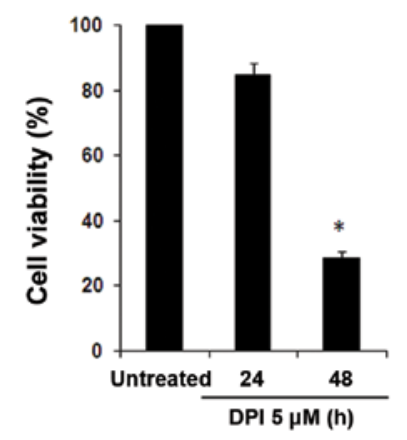

B

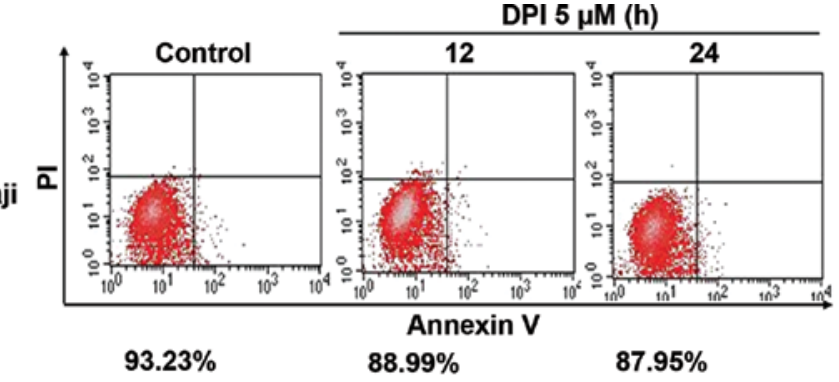

C

Control

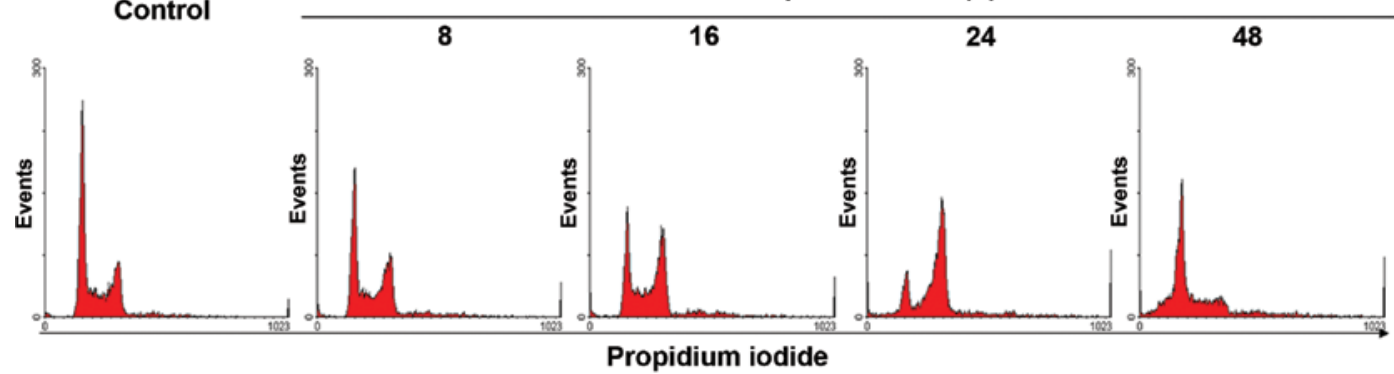

Propidium iodide

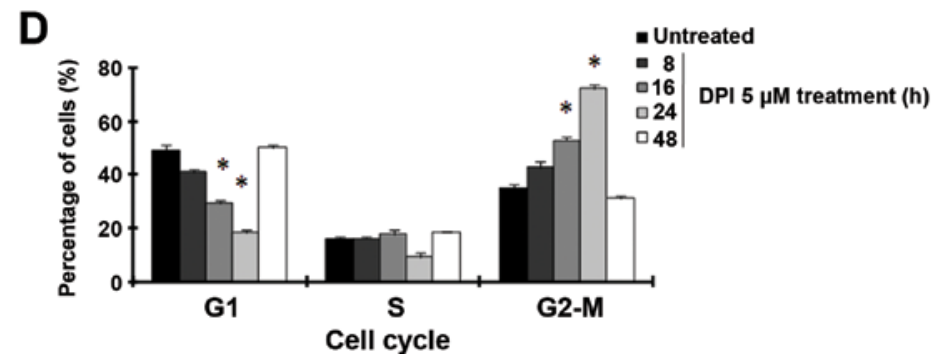

Figure 3. DPI treatment arrests Raji cells at the G2/M phase of the cell cycle. (A) Raji cells were incubated with DPI (5 $\mu$ M) for 0,24 and 48 h, and MTS assays were performed to evaluate the effects of DPI on cell viability (mean \pm SD of three experiments; *p $<0.01$ ). (B) Apoptotic effects of the $5-\mu \mathrm{M}$ DPI treatment on NALM16 and Raji cells at 12 and $24 \mathrm{~h}$ after treatment, as detected by Annexin V-PI staining and flow cytometry. The percentages under the plots indicate live cells, showing both low Annexin V staining and low PI staining. Each histogram is representative of three experiments. (C) Cell cycle analysis of untreated Raji cells, and Raji cells treated with $5 \mu \mathrm{M}$ DPI for 8, 16, 24 and $48 \mathrm{~h}$, as analyzed by flow cytometry and PI staining. Each histogram is representative of three experiments. (D) The effects of DPI treatment on the cell cycle distribution of the Raji cells were analyzed using MultiCycle software program (Phoenix Flow System) (mean \pm SD of three experiments; $\left.{ }^{*} \mathrm{p}<0.01\right)$.

cells, we suspected that DPI may suppress cellular ROS levels in Raji cells by inhibiting NOX activity. As shown in Fig. 2B, flow cytometry indicated that $5 \mu \mathrm{M}$ DPI effectively suppressed ROS accumulation in the Raji cells by blocking NOX activity, causing $\sim 35$ and $57 \%$ decreases in cellular ROS concentrations at 4 and $8 \mathrm{~h}$, respectively. Notably, in addition to suppressing ROS levels, the DPI treatment was able to markedly elevate lactate production in the Raji cells. As shown in Fig. 2C, treatment of Raji cells with $5 \mu \mathrm{M}$ DPI caused significant increases in lactate levels by 2.6- and 5.4-fold at 24 and $48 \mathrm{~h}$, respectively. These increases may have occurred due to upregulation of lactate dehydrogenase (LDH) activity by inhibition of NOX since NOX and LDH utilize the same substrate, NAD $(\mathrm{P}) \mathrm{H}$, to generate ROS and lactate.

DPI treatment arrests Raji cells at the G2-M phase of the cell cycle. Lactate accumulation can be harmful to cancer cells and cause irreversible damage, suggesting that a hyperacidic environment and ROS inhibition may mediate the anticancer effects of DPI. As shown in Fig. 3A, DPI effectively inhibited proliferation of the Raji cells at $48 \mathrm{~h}$, and $\sim 72 \%$ of their capacity for proliferation was inhibited at $5 \mu \mathrm{M}$ DPI. However, both cell viability assays and Annexin V/PI flow cytometric analyses showed that $24 \mathrm{~h}$ of incubation with DPI $(5 \mu \mathrm{M})$ did not cause obvious cell apoptosis in the Raji cells (Fig. 3A and B). Instead of undergoing apoptosis, many of the cells were arrested at the G2-M phase of the cell cycle within $24 \mathrm{~h}$ of DPI treatment (Fig. 3C). Compared to the $34 \%$ of untreated Raji cells at the G2-M phase, 43, 53 and $72 \%$ of these cells were arrested at the G2-M phase after DPI treatments for 8, 16 and $24 \mathrm{~h}$, respectively, as quantified by flow cytometry using PI as a fluorescent probe (Fig. 3C and D). When we prolonged the DPI incubation time to $48 \mathrm{~h}$, this G2-M phase arrest disappeared, and large numbers of Raji cells were arrested at the sub-G1 phase, indicating the occurrence of apoptotic cell death (Fig. 3C and D). These data suggest that the anticancer activity mediated by DPI first resulted in cell cycle arrest during the G2-M phase, which may have led to apoptotic cell death. 
A

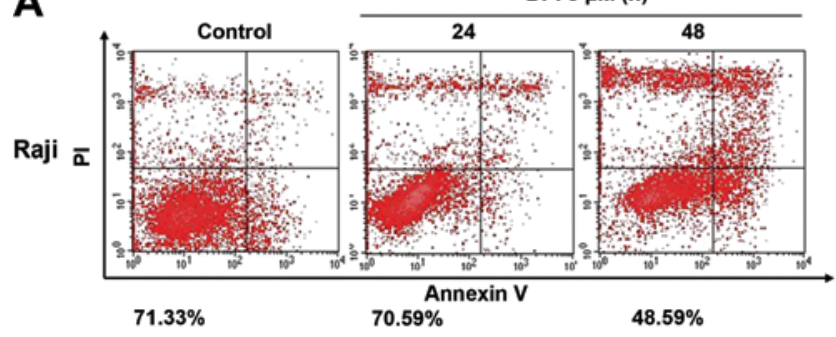

B

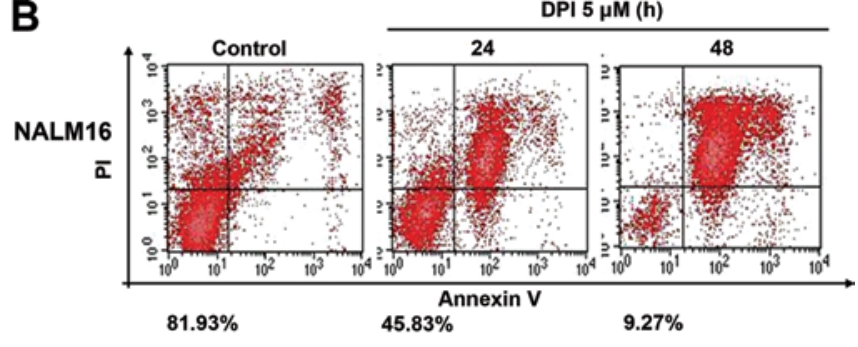

C

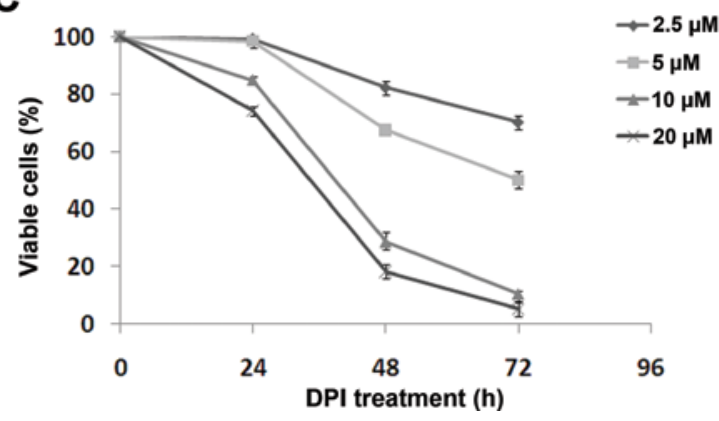

Figure 4. DPI treatment induces apoptosis in Raji BL cells. (A and B) Apoptotic effects of the 5- $\mu$ M DPI treatment on Raji (A) and NALM16 (B) cells at 24 and $48 \mathrm{~h}$ after treatment were detected using Annexin V-PI staining and flow cytometry. The percentages under the plots indicate the live cells with low Annexin V staining and low PI staining. Each histogram is representative of three experiments. (C) Raji cells were treated with 2.5 to $20 \mu \mathrm{M}$ of DPI for 0-72 h. MTS assays were performed to evaluate the effects of DPI on cell viability (mean \pm SD of three experiments).

A

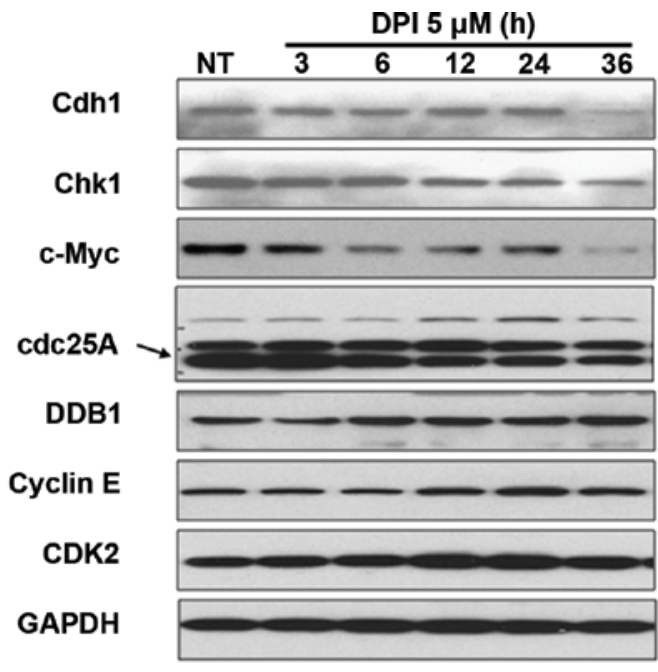

B

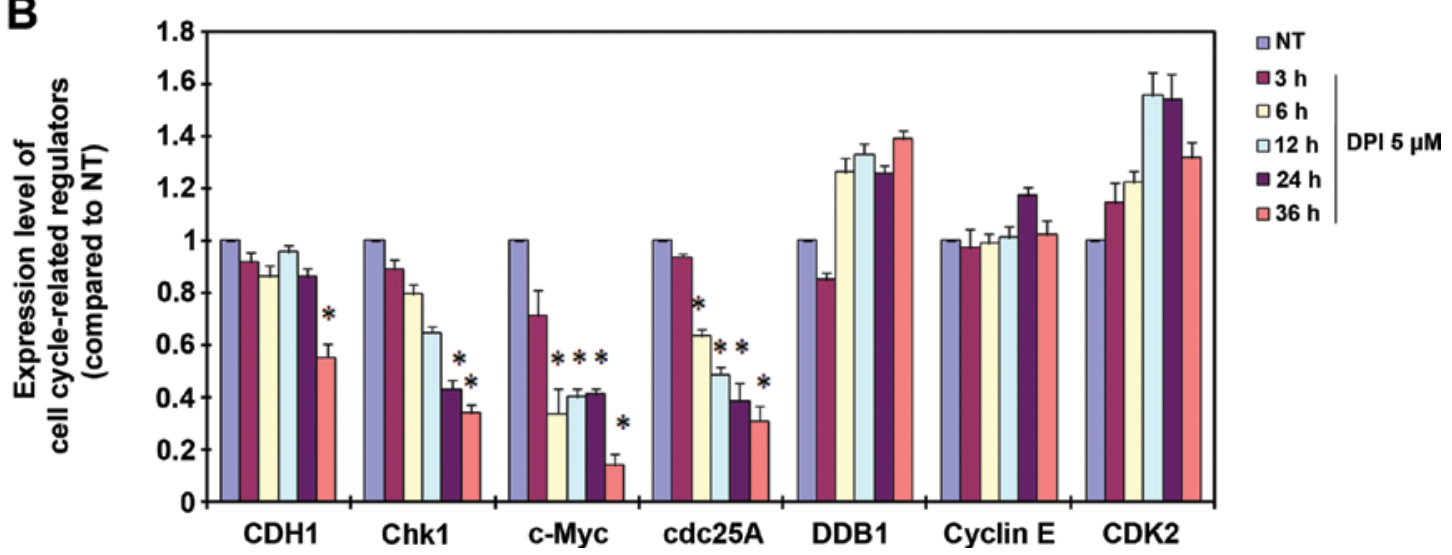

Figure 5. Inhibition of NOX by DPI modifies cell cycle regulators in Raji cells. (A) Immunoblotting analysis was performed to detect the levels of cell cycle regulators in the untreated and 5- $\mu \mathrm{M}$ DPI-treated Raji cells. $\beta$-actin served as a loading control. (B) Analysis of the effects of DPI treatment on the protein expression of the cell cycle regulators, i.e., c-Myc, Cdc25A, Chk1 and Cdh1 (mean \pm SD of three experiments; " $\mathrm{p}<0.01$ ). NOX, NAD(P)H oxidase. 
DPI treatment induces apoptosis in Raji cells. Since the aforementioned flow cytometry data indicated that the apoptotic cell death was the result of DPI-induced cell cycle arrest, we further examined the cell-killing effects of DPI on Raji BL cells. Annexin V/PI flow cytometry assays were adopted to evaluate the cytotoxicity of DPI in the Raji cells. As shown in Fig. 4A and B, compared to the NALM16 cells, which exhibited obvious cell death at both 24 and $48 \mathrm{~h}$ after the 5- $\mu \mathrm{M}$ DPI treatment, Raji cells only exhibited significant apoptotic cell death at $48 \mathrm{~h}$ after the 5- $\mu \mathrm{M}$ DPI treatment, implying a delayed response of these cells to DPI-induced cell cycle arrest. In other words, the DPI-induced destruction of Raji cells may have been caused by the effects of DPI on the cell cycle. Subsequent MTS assays were performed to assess cell viability after DPI treatment at a series of concentrations ranging from 2.5 to $20 \mu \mathrm{M}$. As illustrated in Fig. 4C, the DPI treatment induced time- and dose-dependent cell death in Raji cells, and a significant decrease in cell viability was first observed at $48 \mathrm{~h}$ after treatment. Based on these data, we believe that DPI is a potent killer of Raji BL cells and may represent an improved, novel treatment strategy for the treatment of BL patients.

Cell cycle regulators are involved in DPI-induced cell cycle arrest. By inhibiting NOX activity, DPI arrested the Raji cells at the G2-M phase of the cell cycle and effectively induced apoptotic cell death. To further investigate the related molecular mechanism, a panel of cell cycle regulators was analyzed by immunoblotting following treatments for 3-36 h. The data indicated that the cell cycle regulators Cdh1, Chk1, c-Myc and Cdc25 were modified by the 5- $\mu \mathrm{M}$ DPI treatment (Fig. 5A and B). Among these four proteins, c-Myc and Cdc25 were significantly downregulated at $6 \mathrm{~h}$ after treatment, Chk1 was significantly downregulated at $12 \mathrm{~h}$ after treatment, and Cdh1 was only obviously downregulated at $36 \mathrm{~h}$ after treatment. Based on these modifications of the cell cycle regulators, we suspect that c-Myc and $\mathrm{Cdc} 25$ are quite possibly involved in DPI-induced G2-M cell cycle arrest in the Raji BL cells.

\section{Discussion}

The incidence of BL, which is a highly aggressive form of B lymphoma that remains problematic for children in endemic areas, has increased dramatically worldwide due to its association with immunodeficiency (27). EBV, which is a frequently occurring virus found in all human populations, is strongly linked to the pathogenesis of BL. While most studies have focused on the activities of cancer-related viruses in stimulating the oncogenic signaling pathways of host cells, few investigations have evaluated their roles in modifying the redox status of tumor cells. Recent studies have shown that the hepatitis B virus X protein ( $\mathrm{HBx}$ ) is involved in viral metabolism and ROS accumulation in liver disease (28). Human T-cell leukemia virus type 1 (HTLV-1) has also been demonstrated to stimulate ROS generation under glucose deprivation (29). Previous studies have reported that EBV-positive BL cells express high levels of mitogen-activated protein kinase (MAPK) and have high ROS concentrations compared with EBV-negative malignant B cells (7). In the present study, the role of EBV in ROS stimulation in BL cells and its underlying applications for clinical use were investigated. Following latent EBV infection and LMP1 expression, EBV-positive BL cells (Raji cells) exhibited much higher NOX activity and ROS levels than two EBV-negative malignant B cell lines (JM1 and NALM16). By inhibiting NOX activity, DPI was a potent suppressor of cellular ROS levels, which resulted in lactate accumulation that initially caused significant cell cycle arrest during the G2-M phase and subsequently led to substantial apoptosis of Raji BL cells. Moreover, we suggest that c-Myc and Cdc25A may contribute to DPI-induced cell cycle arrest and the subsequent apoptosis.

EBV infection is known to have three latency programs (latency I, II and III), each of which leads to the production of a distinct panel of viral proteins and RNAs (30). Consistent with a previous report that emphasized the role of EBNA2 and its downstream factor LMP1 in ROS induction in BL cells with EBV type III latent infections (7), our findings suggest an important role of LMP1 in activating NOX and modulating ROS generation in Raji BL cells. When we knocked down LMP1 expression by RNAi, NOX enzyme activity was suppressed.

The NOX family consists of a group of enzymes that utilize molecular oxygen to generate ROS. Since activated NOX enzymes in cancer cells consume the substrate $\mathrm{NAD}(\mathrm{P}) \mathrm{H}$, which is also used as a substrate by LDH to produce lactate, some reports have indicated that NOX is also involved in modulating glycolysis $(31,32)$. Since the activation of NOX enzymes is always linked with inflammation and malignant disorders, it can be targeted to treat these diseases $(33,34)$. DPI is a well-established inhibitor of NOX enzymes. In pancreatic adenocarcinoma cells, DPI potently inhibits ROS production and induces apoptosis (35). In chronic myelogenous leukemia (CML) cells, both DPI and NOX RNAi suppress BCR-ABL-induced cell proliferation and reduce cellular growth (36). In addition to DPI, multiple other compounds, such as plumbagin, celastrol and phenothiazines, have been shown to effectively target NOX (37-39). Here, we demonstrated that inhibition of NOX by DPI not only suppressed ROS levels yet also elevated lactate production. Excessive lactate concentrations can lead to lactic acidosis, causing great harm to cells. In this manner, DPI induced a marked G2-M phase cell cycle arrest that eventually led to irreversible apoptosis in Raji BL cells.

The underlying mechanisms of NOX in cell cycle progression have rarely been investigated, but some information can be obtained from recent publications. ROS produced by NOX enzymes can influence cell cycle progression by regulating phosphorylation and ubiquitination of cyclindependent kinases (CDKs) and cell cycle regulators (40). The cell cycle progression induced by platelet-derived growth factor (PDGF) is partially mediated by NOX1-induced ROS production (26), and NOX4 has been associated with G2-M phase cell cycle progression in melanoma cells (25). In these reports, Cdc 25 phosphorylation and the ubiquitination of CDK inhibitor proteins (CKIs) have been suggested to be involved in NOX-mediated cell cycle progression. In our system, CDK2 expression seemed to be elevated after DPI treatment, possibly due to ubiquitination of CKIs. Consistent with previous findings, Cdc25 expression was altered by DPI treatment. More importantly, c-Myc, which is a vital oncoprotein in BL cells, was significantly downregulated by DPI, strongly implying its potential use for the treatment of BL. 
In summary, the present study linked EBV infection with NOX-activated ROS generation in BL cells, suggesting that NOX activation is a potential target for development of anticancer treatment strategies for BL patients. DPI, which is a specific inhibitor of NOX enzymes, was demonstrated to be capable of decreasing cellular ROS concentrations and of stimulating excess lactate generation, resulting in a G2-M phase cell cycle arrest and eventually leading to significant apoptosis in Raji cells. Moreover, the involved cell cycle regulators were likely c-Myc and Cdc25. Therefore, we propose that EBV-induced NOX activation and ROS generation are potent targets for BL treatment. By halting cell cycle progression and inducing apoptosis, DPI demonstrated the ability to kill Raji BL cells, indicating that it may be valuable for development as an effective anti-BL treatment.

\section{References}

1. Vereide DT, Seto E, Chiu YF, Hayes M, Tagawa T, Grundhoff A, Hammerschmidt W and Sugden B: Epstein-Barr virus maintains lymphomas via its miRNAs. Oncogene 33: 1258-1264, 2014.

2. Bornkamm GW: Epstein-Barr virus and the pathogenesis of Burkitt's lymphoma: more questions than answers. Int J Cancer 124: 1745-1755, 2009.

3. Molyneux EM, Rochford R, Griffin B, Newton R, Jackson G, Menon G, Harrison CJ, Israels T and Bailey S: Burkitt's lymphoma. Lancet 379: 1234-1244, 2012.

4. Drexler HG and Minowada J: History and classification of human leukemia-lymphoma cell lines. Leuk Lymphoma 31: 305-316, 1998.

5. Bieging KT, Amick AC and Longnecker R: Epstein-Barr virus LMP2A bypasses p53 inactivation in a MYC model of lymphomagenesis. Proc Natl Acad Sci USA 106: 17945-17950, 2009

6. Lassoued S, Ben Ameur R, Ayadi W, Gargouri B, Ben Mansour R and Attia $\mathrm{H}$ : Epstein-Barr virus induces an oxidative stress during the early stages of infection in B lymphocytes, epithelial, and lymphoblastoid cell lines. Mol Cell Biochem 313: 179-186, 2008.

7. Cerimele F, Battle T, Lynch R, Frank DA, Murad E, Cohen C, Macaron N, Sixbey J, Smith K, Watnick RS, Eliopoulos A Shehata B and Arbiser JL: Reactive oxygen signaling and MAPK activation distinguish Epstein-Barr Virus (EBV)-positive versus EBV-negative Burkitt's lymphoma. Proc Natl Acad Sci USA 102: 175-179, 2005

8. Zheng H,Li LL, Hu DS, Deng XY and Cao Y: Role of Epstein-Barr virus encoded latent membrane protein 1 in the carcinogenesis of nasopharyngeal carcinoma. Cell Mol Immunol 4: 185-196, 2007.

9. Ha YJ and Lee JR: Role of TNF receptor-associated factor 3 in the CD40 signaling by production of reactive oxygen species through association with $\mathrm{p} 40^{\text {phox }}$, a cytosolic subunit of nicotinamide adenine dinucleotide phosphate oxidase. J Immunol 172 231-239, 2004

10. Lambeth JD, Kawahara T and Diebold B: Regulation of Nox and Duox enzymatic activity and expression. Free Radic Biol Med 43: 319-331, 2007.

11. Devi GS, Prasad MH, Saraswathi I, Raghu D, Rao DN and Reddy PP: Free radicals antioxidant enzymes and lipid peroxidation in different types of leukemias. Clin Chim Acta 293: 53-62, 2000.

12. Park SY, Chang I, Kim JY, Kang SW, Park SH, Singh K and Lee MS: Resistance of mitochondrial DNA-depleted cells against cell death: role of mitochondrial superoxide dismutase. J Biol Chem 279: 7512-7520, 2004.

13. Behrend L, Henderson G and Zwacka RM: Reactive oxygen species in oncogenic transformation. Biochem Soc Trans 31: 1441-1444, 2003.

14. Roth D, Krammer PH and Gulow K: Dynamin related protein 1-dependent mitochondrial fission regulates oxidative signalling in T cells. FEBS Lett 588: 1749-1754, 2014.

15. Trachootham D, Zhou Y, Zhang H, Demizu Y, Chen Z, Pelicano H, Chiao PJ, Achanta G, Arlinghaus RB, Liu J and Huang P: Selective killing of oncogenically transformed cells through a ROS-mediated mechanism by $\beta$-phenylethyl isothiocyanate. Cancer Cell 10: 241-252, 2006.
16. Lin B, Chen Z, Xu Y, Zhang H, Liu J and Qian X: 7-b, a novel amonafide analogue, cause growth inhibition and apoptosis in Raji cells via a ROS-mediated mitochondrial pathway. Leuk Res 35: 646-656, 2011

17. Fengling M, Fenju L, Wanxin W, Lijia Z, Jiandong T, Zu W, Xin Y and Qingxiang G: Rituximab sensitizes a Burkitt lymphoma cell line to cell killing by X-irradiation. Radiat Environ Biophys 48: 371-378, 2009.

18. Kuang Y, Balakrishnan K, Gandhi V and Peng X: Hydrogen peroxide inducible DNA cross-linking agents: targeted anticancer prodrugs. J Am Chem Soc 133: 19278-19281, 2011.

19. Fang J, Nakamura $\mathrm{H}$ and Iyer AK: Tumor-targeted induction of oxystress for cancer therapy. J Drug Target 15: 475-486, 2007.

20. Hu ZY, Wang J, Cheng G, Zhu XF, Huang P, Yang D and Zeng YX: Apogossypolone targets mitochondria and light enhances its anticancer activity by stimulating generation of singlet oxygen and reactive oxygen species. Chin J Cancer 30: 41-53, 2011

21. Acharya A, Das I, Chandhok D and Saha T: Redox regulation in cancer: a double-edged sword with therapeutic potential. Oxid Med Cell Longev 3: 23-34, 2010

22. Lee KW and Lee HJ: The roles of polyphenols in cancer chemoprevention. Biofactors 26: 105-121, 2006.

23. Roy P, George J, Srivastava S, Tyagi S and Shukla Y: Inhibitory effects of tea polyphenols by targeting cyclooxygenase-2 through regulation of nuclear factor kappa B, Akt and p53 in rat mammary tumors. Invest New Drugs 29: 225-231, 2011.

24. Talero E, Ávila-Roman J and Motilva V: Chemoprevention with phytonutrients and microalgae products in chronic inflammation and colon cancer. Curr Pharm Des 18: 3939-3965, 2012.

25. Yamaura M, Mitsushita J, Furuta S, Kiniwa Y, Ashida A, Goto Y, Shang WH, Kubodera M, Kato M, Takata M, Saida T and Kamata T: NADPH oxidase 4 contributes to transformation phenotype of melanoma cells by regulating $\mathrm{G}_{2}-\mathrm{M}$ cell cycle progression. Cancer Res 69: 2647-2654, 2009.

26. Bedard K and Krause KH: The NOX family of ROS-generating NADPH oxidases: physiology and pathophysiology. Physio Rev 87: 245-313, 2007.

27. Mbulaiteye SM, Anderson WF, Ferlay J, Bhatia K, Chang C, Rosenberg PS, Devesa SS and Parkin DM: Pediatric, elderly, and emerging adult-onset peaks in Burkitt's lymphoma incidence diagnosed in four continents, excluding Africa. Am J Hematol 87: $573-578,2012$

28. Gu JM, Lim SO, Oh SJ, Yoon SM, Seong JK and Jung G: HBx modulates iron regulatory protein 1-mediated iron metabolism via reactive oxygen species. Virus Res 133: 167-177, 2008.

29. Silic-Benussi M, Cavallari I, Vajente N, Vidali S, ChiecoBianchi L, Di Lisa F, Saggioro D, D'Ágostino DM and Ciminale V: Redox regulation of T-cell turnover by the p13 protein of human T-cell leukemia virus type 1: distinct effects in primary versus transformed cells. Blood 116: 54-62, 2010.

30. Hutzinger R, Feederle R, Mrazek J, Schiefermeier N, Balwierz PJ, Zavolan M, Polacek N, Delecluse HJ and Hüttenhofer A: Expression and processing of a small nucleolar RNA from the Epstein-Barr virus genome. PLoS Pathog 5: e1000547, 2009.

31. Lu W, Hu Y, Chen G, Chen Z, Zhang H, Wang F, Feng L, Pelicano H, Wang H, Keating MJ, Liu J, McKeehan W, Wang H, Luo Y and Huang P: Novel role of NOX in supporting aerobic glycolysis in cancer cells with mitochondrial dysfunction and as a potential target for cancer therapy. PLoS Biol 10: e1001326, 2012.

32. Prata C, Maraldi T, Fiorentini D, Zambonin L, Hakim G and Landi L: Nox-generated ROS modulate glucose uptake in a leukaemic cell line. Free Radic Res 42: 405-414, 2008.

33. Han CY, Umemoto T, Omer M, Den Hartigh LJ, Chiba T, LeBoeuf R, Buller CL, Sweet IR, Pennathur S, Abel ED and Chait A: NADPH oxidase-derived reactive oxygen species increases expression of monocyte chemotactic factor genes in cultured adipocytes. J Biol Chem 287: 10379-10393, 2012

34. Block K and Gorin Y: Aiding and abetting roles of NOX oxidases in cellular transformation. Nat Rev Cancer 12: 627-637, 2012

35. Mochizuki T, Furuta S, Mitsushita J, Shang WH, Ito M, Yokoo Y, Yamaura M, Ishizone S, Nakayama J, Konagai A, Hirose K, Kiyosawa K and Kamata T: Inhibition of NADPH oxidase 4 activates apoptosis via the AKT/apoptosis signalregulating kinase 1 pathway in pancreatic cancer PANC-1 cells. Oncogene 25: 3699-3707, 2006

36. Singh MM, Irwin ME, Gao Y, Ban K, Shi P, Arlinghaus RB, Amin HM and Chandra J: Inhibition of the NADPH oxidase regulates heme oxygenase 1 expression in chronic myeloid leukemia. Cancer 118: 3433-3445, 2012. 
37. Ding $Y$, Chen ZJ, Liu S, Che D, Vetter $\mathrm{M}$ and Chang $\mathrm{CH}$ Inhibition of Nox-4 activity by plumbagin, a plant-derived bioactive naphthoquinone. J Pharm Pharmacol 57: 111-116, 2005.

38. Gianni D, Taulet N, Zhang H, Der Mardirossian C, Kister J, Martinez L, Roush WR, Brown SJ, Bokoch GM and Rosen H: A novel and specific NADPH oxidase-1 (Nox1) small-molecule inhibitor blocks the formation of functional invadopodia in human colon cancer cells. ACS Chem Biol 5: 981-993, 2010.

39. Jaquet V, Marcoux J, Forest E, Leidal KG, McCormick S, Westermaier Y, Perozzo R, Plastre O, Fioraso-Cartier L, Diebold B, Scapozza L, Nauseef WM, Fieschi F, Krause KH and Bedard K: NADPH oxidase (NOX) isoforms are inhibited by celastrol with a dual mode of action. Br J Pharmacol 164 507-520, 2011.
40. Verbon EH, Post JA and Boonstra J: The influence of reactive oxygen species on cell cycle progression in mammalian cells. Gene 511: 1-6,2012.

41. Pelicano H, Feng L, Zhou Y, Carew JS, Hileman EO, Plunkett W, Keating MJ and Huang P: Inhibition of mitochondrial respiration: a novel strategy to enhance drug-induced apoptosis in human leukemia cells by a reactive oxygen species-mediated mechanism. J Biol Chem 278: 37832-37839, 2003.

42. Guo C, Pan ZG, Li DJ, Yun JP, Zheng MZ, Hu ZY, Cheng LZ and Zeng YX: The expression of p63 is associated with the differential stage in nasopharyngeal carcinoma and EBV infection. $\mathbf{J}$ Transl Med 4: 23, 2006. 